\title{
Error bounds for the modified Newton's method
}

\section{A.L. Andrew}

A strengthened form of the Kantorovich convergence theorem for the modified Newton's method is proved. The result is compared with previously known results.

\section{Introduction}

Let $F$ be a continuously Fréchet differentiable mapping of an open subset $\Omega$ of a Banach space $X$ into a Banach space $Y$. This note concerns the numerical solution of

$$
F(x)=0
$$

by the modified Newton's method, that is by the iteration

$$
x_{n+1}=x_{n}-\Gamma_{0} F\left(x_{n}\right) \text {, }
$$

where $\Gamma_{0}=\left[F^{\prime}\left(x_{0}\right)\right]^{-1}$, the inverse of the Fréchet derivative of $F$ at $x_{0}$. Dennis [1] has strengthened Kantorovich's convergence theorem [2, Theorem 6 (1.XVIII)] for both (2) and the original Newton's method.

Theorem 1 below further strengthens Dennis's result for (2). It proves existence and local uniqueness of the solution of ( 1 ) and the convergence of (2) to this solution under weaker conditions on $F$, and for a given $F$ establishes a rate of convergence for (2) which is at least as fast as, and generally faster than, that proved by Dennis. Ways in which Theorem 1 may be further strengthened are noted. Theorem 1 may be proved by modifying Dennis's proof, but in this case the proof given below, which follows [2] even more closely, is simpler. For further references, see [3].

Received 1 March 1976. 


\section{Error bounds}

THEOREM 1. Let $\Omega_{0}=\left\{x:\left\|x-x_{0}\right\| \leq r\right\} \subset \Omega$. Let $\Gamma_{0}$ exist in $L(Y, X)$, the set of bounded linear mappings from $Y$ into $X$, and let $n=\left\|\Gamma_{0} F\left(x_{0}\right)\right\|$. Let

$$
\left\|I-\Gamma_{0} F^{\prime}(x)\right\| \leq K\left\|x_{0}-x\right\| \text { for alz } x \text { in } \Omega_{0},
$$

where $I$ is the identity operator. Let $0<h=K \eta \leq \frac{7}{2}$ and $r \geq r$. where

$$
r_{ \pm}=\left[1 \pm(1-2 h)^{\frac{3}{2}}\right] n / h \text {. }
$$

Then all elements of the sequence defined by (2) lie in $\Omega_{0}$, and the sequence converges to a solution $x^{*}$ of (1) with

$$
\left\|x^{*}-x_{n}\right\| \leq \frac{\eta}{2 h}\left[1-(1-2 h)^{\frac{1}{2}}\right]^{n+1}, n=1,2, \ldots .
$$

The inequality is strict if $n>1$. If also either $r<r_{+}$or $r_{-}=r=r_{+}$, then $x^{*}$ is the only solution of (1) in $\Omega_{0}$.

Note that if $h=0$, then either (1) is linear or $F\left(x_{0}\right)=0$, so that in neither case does the question of convergence arise. The proof of Theorem 1 uses the following two lemmas.

LEMMA 1. Let $\psi \in C^{l}[0, r]$ be a real valued fronction with $c_{0}=\left[-\psi^{\prime}(0)\right]^{-1}>0$ and $1+c_{0} \psi^{\prime}(t) \geq 0$ for all $t \geq 0$. Let

$$
\psi(t)=0
$$

have a root in the interval $[0, r]$ and let $t^{*}$ be the smallest root of (5) in $[0, r]$. Let $\Gamma_{0} \in L(Y, X)$ and, for some nonnegative integer $p$, Let

(i) $\left\|\Gamma_{0} F\left(x_{i}\right)\right\| \leq c_{0} \psi\left(t_{i}\right), \quad i=0, \ldots, p$, and

(ii) $\left\|I-\Gamma_{0} F^{\prime}(x)\right\| \leq 1+c_{0} \psi^{\prime}(t)$ whenever

$$
\left\|x-x_{p}\right\| \leq t-t_{p} \leq r-t_{p},
$$

where $t_{0}=0$ and $t_{n+1}=t_{n}-\psi\left(t_{n}\right) / \psi^{\prime}\left(t_{0}\right)$, 


$$
n=0,1, \ldots .
$$

Then (1) has a solution $x^{*}$ in $\Omega_{p}=\left\{x:\left\|x-x_{p}\right\| \leq r-t_{p}\right\}$, all members of the sequence defined by (2) lie in $\Omega_{0}$, and

$$
\left\|x^{*}-x_{n}\right\| \leq t^{*}-t_{n}, n=0,1, \ldots .
$$

If also $\psi^{\prime}(r) \leq 0$ and (5) has a vonique solution in $[0, r]$ then (1) has a vonique solution in $\Omega_{p}$.

The proof is omitted since it requires only minor changes in the proofs of Theorems $1,2,3$, and 4 (I.XVIII) of [2]. Note that $\Omega_{p+1} \subset \Omega_{p}$ and the proof of Theorem 1 (1.XVIII) of [2] shows that if conditions ( $i$ ) and $(i i)$ of Lemma 1 are satisfied for some integer $p$, they are also satisfied for $p+1$.

LEMMA 2. Let $f(t)=K t^{2}-2 t+2 \eta$ where $K, \eta$ are the constants defined in Theorem 1 and $0<h=K \eta \leq \frac{1}{2}$. Let $t_{0}=0$ and $t_{n+1}=t_{n}-f\left(t_{n}\right) / f^{\prime}\left(t_{0}\right), n=0,1, \ldots$. Then $t_{n} \rightarrow r_{-}$as $n \rightarrow \infty$ and, for $n \geq 1, t_{n-1}<t_{n}<r_{-}$and

$$
r_{-}-t_{n}=r_{-} \prod_{i=0}^{n-1} \frac{K\left(r_{-}+t_{i}\right)}{2} \leq \frac{\eta}{2 h}\left[1-(1-2 h)^{\frac{1}{2}}\right]^{n+1} \text {, }
$$

with strict inequality in (6) when $n>1$.

The proof, which uses the fact that $f\left(r_{ \pm}\right)=0$, is by induction.

It is readily verified that $f$ has the properties required of $\psi$ in Lemma 1 with $r_{-}=t^{*}$ so that Theorem 1 follows.

The proof of Theorem 1 uses only the case $p=0$ in Lemma 1 . The case $p=1$ yields the following result.

THEOREM 2. Let all the conditions of Theorem 1 be satisfied except that $K$ is not required to satisfy (3). Let $\left\|\Gamma_{0} F\left(x_{1}\right)\right\| \leq K n^{2} / 2$ and let

$$
\left\|I-\Gamma_{0} F^{\prime}(x)\right\| \leq K\left(\left\|x-x_{1}\right\|+\eta\right) \text { for all } x \text { in } \Omega_{1} \text {. }
$$

Then all the conclusions of Theorem 1 follow except possibly the uniqueness in $\Omega_{0}$ of the solution of (1). The solution is however conique in $\Omega_{1}$. 
The proofs of Theorems 1 and 2 show that sharper but more complicated bounds for $\left\|x^{*}-x_{n}\right\|$ may easily be obtained by using the equality instead of the inequality in (6).

\section{Comparison with previously known results}

Theorem 1 strengthens Corollary 4.1 of [1] in two respects. The result in [1] proves only weak inequality in (4) and, more importantly, it replaces (3) with the stronger condition

$$
\left\|\Gamma_{0} F^{\prime}(x)-\Gamma_{0} F^{\prime}(y)\right\| \leq K\|x-y\| \text { for all } x \text { and } y \text { in } \Omega_{0} \text {. }
$$

Otherwise the results are identical.

Let $h_{1}, h_{2}, h_{3}$ be the smallest possible values of $h=K n$ when $K$ is defined as in Theorem 1, Theorem 2, and (7), respectively. Clearly $h_{3} \geq h_{1}$, and the one dimensional example

$$
F(x)=x-1+a \sin e^{b x}, x_{0}=0,
$$

with $a$ and $b$ constants, $b$ large and $a$ very small, shows that the ratio $h_{3} / h_{1}$ may be arbitrarily large. Also the remark following Lemma 1 shows that $h_{1} \geq h_{2}$ and the one dimensional example

$$
F(x)=x-1+c^{-1} \cos (c x / 5), x_{0}=0,
$$

where $c=10 n \pi+1$ and $n$ is a large integer, shows that $h_{1} / h_{2}$ may be arbitrarily large. Since $r_{-}$increases with $h$ in $\left(0, \frac{1}{2}\right)$, it is clear that decreasing $h$ in Theorem 1 sharpens the error bounds (4) and weakens the conditions required for convergence. Moreover it is sometimes easier to calculate $h_{1}$ or $h_{2}$, or upper bounds for them that are smaller than $h_{3}$, than it is to calculate $h_{3}$. As shown in [1], Kantorovich's result is still weaker than the results in [1].

As well as giving a priori error bounds, Theorem 1 may also be used to calculate more accurate $a$ posteriori error bounds. Let $y$ be an approximation to $x^{*}$ obtained by (2) or by any other means. Set $x_{0}=y$ and calculate $x_{1}$ by (2). Then (4) with $n=1$ gives an error bound for 
$x_{1}$. Generally this will be better than can be obtained from known bounds for Newton's method as such bounds involve $h_{3}$ instead of $h_{1}$. However since the difference between $h_{3}$ and $h_{1}$ is generally smaller when $x_{0}$ is nearer $x^{*}$, the gain from using (4) will be less than is the case with a priori bounds.

Both a priori and a posteriori error bounds for (2) are sometimes obtained from the following special case of the contraction mapping theorem $[2,4]$.

THEOREM 3. Using the same notation as before, let $\Gamma_{0}$ exist and let

$$
\alpha=\sup _{x \in \Omega_{0}}\left\|I-\Gamma_{0} F^{\prime}(x)\right\|<1,
$$

where $\Omega_{0} \subset \Omega$ and $r=n /(1-\alpha)$. Then (1) has a vonique solution $x^{*}$ in $\Omega_{0}$, and, for $n \geq 1, x_{n} \in \Omega_{0}$ and

$$
\left\|x_{n}-x^{*}\right\| \leq \frac{\alpha}{1-\alpha}\left\|x_{n}-x_{n-1}\right\| \leq \frac{\alpha^{n}}{1-\alpha}\left\|x_{1}-x_{0}\right\|=\frac{\alpha^{n} \eta}{1-\alpha}
$$

Note that each of Theorems 1,2, and 3 proves convergence of (2) for (8) when, say, $a=10^{-4}, b=5$, al though the theorems of Kantorovich and Dennis both fail in this case.

In their important book on shooting methods, Roberts and Shipman [4, p. 126] showed that if $F \in C^{2}\left(\Omega_{0}\right)$, then

$$
\alpha^{2}-\alpha+h_{4} \geq 0
$$

whenever $h_{4} \geq\left\|\Gamma_{0} F^{\prime \prime}(x)\right\| \eta$ for all $x$ in $\Omega_{0}$. Clearly $h_{4} \geq h_{3} \geq h_{1}$. From (9) they deduced, erroneously as (8) shows, that

$$
\alpha \geq\left[1-\left(1-4 h_{4}\right)^{\frac{7}{2}}\right] / 2 \text {, }
$$

and hence that Kantorovich's Theorem always gave sharper error bounds than does Theorem 3. In fact the one dimensional example

$$
F(x)=10-x+c\left[\min \left(0, x^{2}-1\right)\right]^{2}, x_{0}=0,
$$


where $c$ is a constant, shows that sometimes Theorem 3 also gives sharper error bounds than Theorem 1. However Theorem 4 below shows that for an important class of functions Theorem 1 gives sharper error bounds than Theorem 3. For many, but not all, of these functions, Kantorovich's Theorem also proves sharper error bounds than Theorem 3 .

THEOREM 4. Let $F$ have the properties required in Theorems 1 and 3 and in addition let the maximm value of

$$
\left\|I-\Gamma_{0} F^{\prime}(x)\right\| /\left\|x_{0}-x\right\|
$$

for $x$ satisfying $0<\left\|x_{0}-x\right\| \leq r$ be attained on the boundary $\left\|x_{0}-x\right\|=r$. Let the bounds for $\left\|x^{*}-x_{n}\right\|$ given by Theorem 1 with $h=h_{1}$ and by Theorem 3 be $A_{n}$ and $B_{n}$ respectively. Then Theorem 3 proves convergence of (2) only if $h_{1} \leq \frac{1}{4}$. Also $B_{n} / A_{n}$ increases with $h$ and $n$ and is always greater than 2 .

Proof. A simplification of the proof of (9) [4] shows that in this case

$$
\alpha^{2}-\alpha+h_{1}=0
$$

It follows that, since $\alpha$ must be real, Theorem 3 is applicable only when $h_{1} \leq \frac{3}{4}$ and that in this case

$$
\alpha \geq\left[1-\left(1-4 h_{1}\right)^{\frac{7}{2}}\right] / 2
$$

Hence for $0<h_{1} \leq \frac{3}{4}$ and $n \geq 1$,

$$
\begin{aligned}
\frac{B_{n}}{A_{n}} & \geq \frac{4 h_{1}}{\left[1+\left(1-4 h_{1}\right)^{\frac{1}{2}}\right]\left[1-\left(1-2 h_{1}\right)^{\frac{1}{2}}\right]}\left[\frac{1-\left(1-4 h_{1}\right)^{\frac{1}{2}}}{2\left[1-\left(1-2 h_{1}\right)^{\frac{1}{2}}\right]}\right]^{n} \\
& =2\left[g\left(h_{1}\right)\right]^{n+1},
\end{aligned}
$$

where $g(h)=\left[1+(1-2 h)^{\frac{1}{2}}\right] /\left[1+(1-4 h)^{\frac{1}{2}}\right]$. Clearly $g(h)>1$ and $g^{\prime}(h)>0$ for $0<h \leq \frac{1}{4}$. The result follows. 


\section{References}

[1] J.E. Dennis, Jr., "On the Kantorovich hypothesis for Newton's method", SIAM J. Numer. Anal. 6 (1969), 493-507.

[2] L.V. Kantorovich and G.P. Akilov, Functional analysis in normed spaces (translated by D.E. Brown. International Series of Monographs in Pure and Applied Mathematics, 46. Pergamon Press, Oxford, London, Edinburgh, New York, Paris, Frankfurt, 1964).

[3] J.M. Ortega and W.C. Rheinboldt, Iterative solution of nonlinear equations in several variables (Academic Press, New York and London, 1970).

[4] Sanford M. Roberts, Jerome S. Shipman, Two-point boundary value problems: shooting methods (Modern Analytic and Computational Methods in Science and Mathematics, 31. American Elsevier, New York, 1972).

Department of Mathematics,

La Trobe University,

Bundoora,

victoria. 